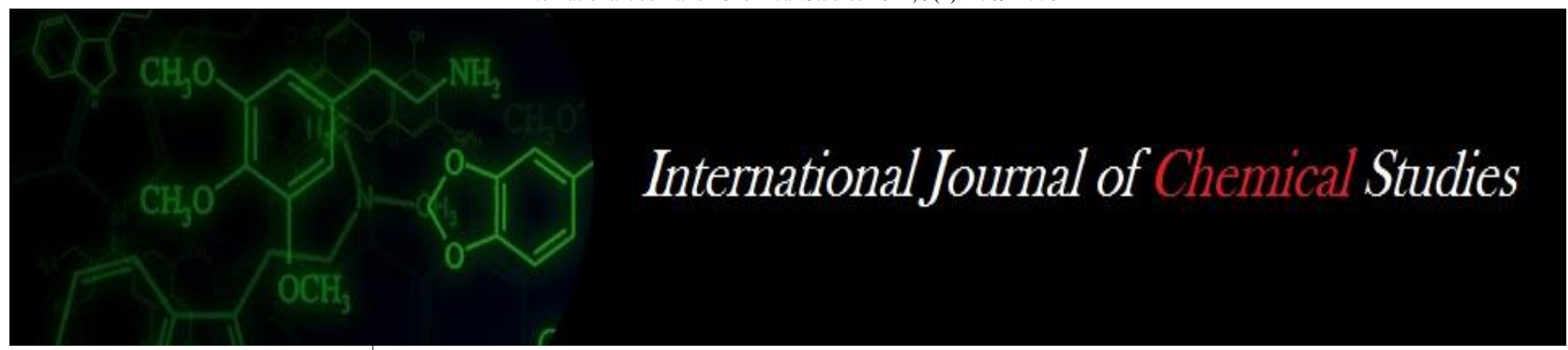

P-ISSN: 2349-8528

E-ISSN: 2321-4902

www.chemijournal.com

IJCS 2021; 9(1): 1769-1776

(C) 2021 IJCS

Received: 01-10-2020

Accepted: 10-12-2020

\section{P Sridevi}

Agricultural College, Bapatla,

Andhra Pradesh, India

B Krishna Veni

Agricultural Research Station,

Bapatla, Andhra Pradesh, India

D Sandeep Raja

Post-Harvest Technology Centre,

Bapatla, Andhra Pradesh, India

DPB Jyothula

Agricultural College, Bapatla,

Andhra Pradesh, India
Corresponding Author:

B Krishna Veni

Agricultural Research Station,

Bapatla, Andhra Pradesh, India

\section{Physico-chemical, nutritional and Anti-oxidative properties of different colored grain genotypes of rice (Oryza sativa $\mathbf{L}$.)}

\author{
P Sridevi, B Krishna Veni, D Sandeep Raja and DPB Jyothula
}

DOI: https://doi.org/10.22271/chemi.2021.v9.i1y.11482

\begin{abstract}
The commonly consumed white rice is a highly refined staple cereal, which is lack of almost all minerals and nutrients. The pigmented rice, enriched with anthocyanin is known for it's taste and health benefits. Colored rice (Oryza sativa L) genotypes are evaluated under this study. Twenty six genotypes were studied for 8 yield components and 12 physico-chemical, nutritional and Anti-oxidative properties. Among the non-pigmented varieties, BPT 5204, BPT 2270, BPT 2595, BPT 2782 and BPT 2776 recorded the desirable quality traits with excellent cooking quality. The pigmented rice genotypes recorded high amount of total phenols, antioxidant activity and flavonoid contents than non-pigmented varieties. When compared with light brown pericarp colored rice genotypes, red and black rice genotypes possess more protein content, high $\mathrm{Zn}$ and Fe content also. Variability studies showing that, high GCV and PCV coupled with high heritability and high genetic advance as percent of mean were recorded for test weight and total number of grains per panicle among yield components, while among quality traits solid loss, alkali spreading value, total phenol content, total antioxidant activity, flavonoid content, zinc and iron content. It indicates that these characters are controlled by additive type of gene action, which can be improved by direct selection. The results of correlation studies revealed that total phenol content, flavonoid content, total antioxidant activity are positively associated with each other and also with protein content, $\mathrm{Zn}$ and $\mathrm{Fe}$ content, hence simultaneous improvement of all these traits is anticipated. The colored rice varieties reported in the present study recorded medium duration, high yield potential coupled with medium slender grain type and good cooking quality and may be exploited commercially. Black and red rice genotypes viz., BPT 2848, BPT 3140, BPT 3141, BPT 2858, BPT 3111 which recorded desirable physical quality traits, high antioxidant activity, high protein, Fe \& Zn content can be included in daily diet for their potential bioactive compounds and neutraceutical benefits to human health.
\end{abstract}

Keywords: Colored rice, total phenol content, antioxidant activity, protein content

\section{Introduction}

Rice (Oryza sativa L.) is the predominant staple food crop for more than half of the world's population and is playing a pivotal role in providing human nutrition, energy supply and food security of Asian countries. Rice is not only the major source of energy, but also the major source of protein and a significant source of fiber and essential micronutrients (Uphoff, 2008) [46]. Rice is the only cereal, cooked and consumed as a whole grain and quality considerations are much more important than for any other food crops (Hossain et al.,. 2009) ${ }^{[9]}$. Nowadays, whole grain pigmented rice has been categorized as one of the potent functional foods. The total antioxidant activity which is positively correlated with the total soluble phenolic compounds (TSPCs) are found in higher concentration in genotypes with red and black pericarp color when compared to those with a light brown pericarp color (Tian et al., 2004) ${ }^{[44]}$. Black rice powder extracted from pigmented rice bran could be used as a natural food coloring dye in the preparation of cosmetics, sodas, functional foods, nutraceutical and other healthy food products. Black and red rice bran can be an excellent ingredient to increase the nutritional value and antioxidant properties of noodles (Kong et al., 2012) ${ }^{[18]}$. Recently, rice consumers are showing great interest for colored rice varieties due to their potential health benefits. Hence, the present study was undertaken with an objective to estimate the nutritional and antioxidative properties of different colored rice genotypes and to identify rice varieties with high neutraceutical value. 


\section{Materials and Methods \\ Rice samples}

Twenty six rice (Oryza sativa L.) genotypes, consisting of 7 non-pigmented (brown pericarp colored) rice (BPT 5204, BPT 2270, BPT 2295, BPT 2782, BPT 2595, BPT 2660 and BPT 2776), nine genotypes possessing red pericarp (Aanthra, Samyuktha, Matha Triveni, Jyothi, Annapurna, Harsha, BPT 3111, BPT 3139 and BPT 2858) and 10 genotypes with black pericarp (BPT 3136, BPT 3137, BPT 3138, BPT 3140, BPT 3141, BPT 3142, BPT 3143, BPT 3144, BPT 3145 and BPT 2848) were grown during Kharif, 2018 at Agricultural College Farm, Bapatla, Andhra Pradesh. Among these, BPT 5204, BPT 2270, BPT 2295, BPT 2595 and BPT 2782 varieties were released in Andhra Pradesh while red rice varieties viz. Matha Triveni, Annapurna, Aathira, Harsha, Jyothi and Samyuktha were released in Kerala state. All other genotypes used in the study are advanced cultures developed at Agril. Research Station, Bapatla, Andhra Pradesh, India. Each genotype was sown under dry direct sowing method in 5 rows of $3 \mathrm{~m}$ length in Randomized Block Design with three replications. The crop was raised by following standard package of practices and observations were taken on 5 plants per replication for eight morphological characters viz., days to $50 \%$ flowering, plant height, number of productive tillers, panicle length, test weight, total number of grains/panicle, fertility percentage and grain yield/plant. After harvesting, the paddy was sun-dried to a moisture content of about $12 \%$, stored in an air-tight plastic bag at room temperature for three months and then stored at $4{ }^{\circ} \mathrm{C}$ in the dark before analysis. Each sample per genotype per replication (three replicates per genotype) was dehusked and the unpolished rice was utilized for estimation of 12 physico-chemical, nutritional and Antioxidative properties during the year 2019. The calculation of $\mathrm{L} / \mathrm{B}$ ratio and classification of grain type were carried out as per IIRR (2006) ${ }^{[10]}$.

Determination of total phenol content (TPC): TPC was estimated by using Folin-Ciocalteu (FC) reagent (Malik C.P and Singh M.B. 1980). The results were expressed as milligrams of gallic acid equivalent per 100 gram of sample (mg GAE/ $100 \mathrm{~g}$ ).

Determination of Total Antioxidant Activity: The total antioxidant activity was measured using DPPH radical scavenging as described by Pathirana et al. (2005) ${ }^{[30]}$. The result was expressed as milligram of ascorbic acid equivalent per 100 gram of sample (mg AAE/100g).

Determination of total flavonoid content (TFC): TFC was estimated as suggested by Swain et al. (1959) The results were expressed as milligrams of catechin equivalent per $100 \mathrm{~g}$ of dry rice flour ( $\mathrm{mg} \mathrm{CE} / 100 \mathrm{~g}$ ).

Amylose content: The amylose content in test samples were estimated by following the method delineated by Juliano $(1971)^{[14]}$ and expressed as percentage.

Alkali spreading value: The alkali spreading value of the rice kernels were noted on a seven point scale and expressed as average of six values. Scoring was done by following the method described by Little et al. (1958) ${ }^{[23]}$.

Volume Expansion Ratio and Solid loss: Volume expansion ratio and the amount of total solid lost in cooking water was determined as per Sidhu et al. (1975) ${ }^{[39]}$.
Protein content: The protein content was estimated by using Lowry's method (Lowry et al., 1951) ${ }^{[24]}$. Protein content was calculated by comparing the O.D. values of the standard with test sample and expressed as percentage.

Zn content (ppm), Iron content (ppm): The $\mathrm{Zn}$ and $\mathrm{Fe}$ content was estimated by using the energy dispersive $\mathrm{X}$ - ray Fluorescence Spectroscopy (XRF) by non destructive method at Indian Institute of Rice Research, Hyderabad (IIRR, 2006) ${ }^{[10]}$.

\section{Statistical analysis}

Statistical analyses of experimental data were analyzed using the statistical software package of SAS 9.2 software. The data in triplicate for all the parameters was given as Mean \pm SD. All comparisons were subjected to two way analysis of variance (ANOVA) and statistical significance was set at $p<0.05$. The genotypic and phenotypic correlation analysis was performed with Pearson's correlation test and statistical significance were reported at $p<0.01$.

\section{Results and Discussion}

Mean, variability (GCV, PCV), Heritability and Genetic Advance (as percent of mean)

Above genetic parameters were studied for all the characters (Table 1). The study reveals that:

Among the yield component traits test weight (PCV-22.18\% GCV-21.87\% Heritability-97.2\% GAM-44.41) and total number of grains per panicle (PCV-24.2\% GCV-20.69\% Heritability-73.10\% GAM-36.45) recorded high estimates for all the genetic parameters studied. These results are in agreement with the findings reported by Chowdhury et al. (2016) ${ }^{[3]}$ and Sameera et al. (2016) ${ }^{[35]}$. While grain yield (Heritability-77.10\% GAM-28.98) recorded high estimates for heritability and genetic advance (as percent of mean). It is in accordance with results reported by Chowdhury et al. $(2016)^{[3]}$.

Among physical and cooking quality parameters, the traits viz., solid loss (PCV-34.59\% GCV-34.46\% Heritability99.20\% GAM-70.70), alkali spreading value (PCV-28.77\% GCV-24.01\% Heritability-69.70\% GAM-41.29), volume expansion ratio (Heritability-86.40\% GAM-34.43) and amylose content (Heritability-93\% GAM-21.89) manifested high estimates for the genetic parameters studied. These results are in accordance with the findings of Lakshmi et al. (2017) ${ }^{[19]}$ and Mamata et al. (2018) ${ }^{[26]}$.

Among nutritional quality parameters, total phenol content (PCV-43.23\% GCV-43.13\% Heritability-99.76\% GAM88.85), total antioxidant activity(PCV-38.92\% GCV-38.82\% Heritability-99.50\% GAM-79.77), flavonoid content(PCV$59.12 \%$ GCV-59.03\% Heritability-99.70\% GAM-121.41), zinc content(PCV-21.73\% GCV-20.47\% Heritability-88.70\% GAM-39.71), iron content(PCV-27.38\% GCV-23.12\% Heritability-71.30\% GAM-40.21), protein content (Heritability-99.40\% GAM-48.48), exhibited high estimates for the genetic parameters studied. These findings are in accordance with results reported by Devi et al. (2016) ${ }^{[4]}$, Raja et al. (2013) ${ }^{[34]}$ and Haritha et al. (2020) ${ }^{[7]}$.

Above results suggesting that inheritance of these traits is controlled by additive gene effects. Which is fixable, it is indicating that, these characters can be improved by direct selection. The remaining traits viz., ear bearing tillers, plant height, panicle length and days to $50 \%$ flowering, L/B ratio are controlled by both additive and non additive gene effects and the improvement of these traits may not be anticipated through simple selection. 
Table 1: Mean, variability, heritability and genetic advance as per cent of mean for yield, yield components and grain quality parameters in rice (Oryza sativa L.)

\begin{tabular}{|c|c|c|c|c|c|c|c|c|}
\hline \multirow{2}{*}{$\begin{array}{c}\text { S. } \\
\text { No. }\end{array}$} & \multirow{2}{*}{ Character } & \multirow[t]{2}{*}{ Mean } & \multicolumn{2}{|c|}{ Range } & \multicolumn{2}{|c|}{$\begin{array}{l}\text { Coefficient of } \\
\text { variation }\end{array}$} & \multirow{2}{*}{$\begin{array}{l}\text { Heritability } \\
\text { (\%) (broad } \\
\text { sense) }\end{array}$} & \multirow{2}{*}{$\begin{array}{l}\text { Genetic advance as } \\
\text { per cent of mean (5\% } \\
\text { level) }\end{array}$} \\
\hline & & & Minimum & Maximum & PCV \% & GCV \% & & \\
\hline 1 & Days to $50 \%$ flowering & 108.52 & 103.00 & 127.00 & 8.70 & 8.12 & 87.00 & 15.60 \\
\hline 2 & Panicle length (cm) & 27.14 & 19.89 & 32.44 & 11.26 & 9.41 & 69.80 & 16.19 \\
\hline 3 & Plant height $(\mathrm{cm})$ & 111.37 & 88.40 & 123.53 & 7.34 & 6.65 & 82.00 & 12.41 \\
\hline 4 & Ear bearing tillers per plant & 14.67 & 12.33 & 18.33 & 14.91 & 8.99 & 36.40 & 11.18 \\
\hline 5 & Grain Yield per plant $(\mathrm{g})$ & 41.09 & 25.00 & 58.33 & 18.24 & 16.02 & 77.10 & 28.98 \\
\hline 6 & Test Weight $(\mathrm{g})$ & 18.51 & 13.49 & 27.90 & 22.18 & 21.87 & 97.20 & 44.41 \\
\hline 7 & Total number of grains per panicle & 298.78 & 191.00 & 410.66 & 24.20 & 20.69 & 73.10 & 36.45 \\
\hline 8 & Fertility $\%$ & 91.41 & 85.93 & 95.58 & 3.61 & 2.11 & 34.30 & 2.55 \\
\hline 9 & Solid Loss & 1.51 & 0.60 & 2.75 & 34.59 & 34.46 & 99.20 & 70.70 \\
\hline 10 & Volume Expansion Ratio & 2.21 & 1.50 & 3.25 & 19.35 & 17.98 & 86.40 & 34.43 \\
\hline 11 & Alkali Spreading Value & 3.42 & 2.00 & 5.00 & 28.77 & 24.01 & 69.7 & 41.29 \\
\hline 12 & L/B ratio & 2.80 & 2.26 & 3.64 & 14.27 & 10.56 & 54.80 & 16.10 \\
\hline 13 & Amylose Content (\%) & 22.42 & 16.41 & 27.44 & 11.43 & 11.02 & 93.0 & 21.89 \\
\hline 14 & Protein Content $(\%)$ & 10.59 & 6.23 & 13.55 & 6.21 & 6.18 & 99.40 & 48.48 \\
\hline 15 & Total Phenol Content (mg/100 g) & 96.10 & 48.80 & 214.34 & 43.23 & 43.13 & 99.76 & 88.85 \\
\hline 16 & Total Antioxidant Activity (mg AAE/100 g) & 78.61 & 26.87 & 111.48 & 38.92 & 38.82 & 99.50 & 79.77 \\
\hline 17 & Flavonoid Content $(\mathrm{mg} / 100 \mathrm{~g})$ & 349.55 & 82.55 & 784.54 & 59.12 & 59.03 & 99.70 & 121.41 \\
\hline 18 & Zn content $(\mathrm{ppm})$ & 21.73 & 12.00 & 27.90 & 21.73 & 20.47 & 88.70 & 39.71 \\
\hline 19 & Iron content (ppm) & 10.63 & 7.00 & 19.40 & 27.38 & 23.12 & 71.30 & 40.21 \\
\hline
\end{tabular}

PCV = phenotypic coefficient of variation in \%; GCV = genotypic coefficient of variation in \%;

PCV and GCV categories: 0-10\% low; 10-20\% medium; > 20\% high (Siva Subramanian and Menon, 1973)

Heritability categories: 0-30\% low; 30-60\% moderate; >60\% high (Johnson et al. 1955)

GAM categories: $0-10 \%$ low; 10-20\% moderate; >20\% high (Johnson et al. 1955)

\section{Grain Yield and Physico-chemical quality traits of different colored rice genotypes}

Maximum grain yield/plant was recorded in black pericarp colored genotype BPT $3142(58.33 \mathrm{~g})$ followed by Matha Triveni (red rice) and BPT 3136 (black rice). Among the non pigmented rice genotypes, BPT $2782(47.7 \mathrm{~g})$ followed by BPT 2776 (40.33g) exhibited high grain yield/plant (Table 2). Due to increased health consciousness and demand for colored rice, farmers in Andhra Pradesh are growing black rice varieties like Burma black, Kalabhatt and Chakhao amudi which are popular in North eastern India. But these varieties are tall in stature, low yielding, possess bold grain and had low amylose content which are not preferred by the consumers in South India. Purwanto et al. (2018) ${ }^{[33]}$ stated that only few farmers were willing to cultivate black rice, because of their longer duration and low grain yield/plant (16.8- $23.94 \mathrm{~g}$ grain weight/plant). Somsana et al. (2013) ${ }^{[40]}$ also reported that farmers of Nepal mostly grow local genotypes of black glutinous rice with low yield potential. The black glutinous rice is mainly used for the preparation of sweet snacks and desserts in Asia which may not be included in daily diet, particularly in South India where rice consumer does not prefer the sticky nature of cooked rice. The colored rice genotypes reported in the present investigation had medium duration (102-108 days for 50\% flowering), high yield potential (34.9-58.3g/plant) with medium slender grain type coupled with good cooking quality may be exploited commercially. Among the genotypes, non pigmented rice varieties possessed medium slender grain type which is preferred by the consumers of South India (Table 3). Except Matha Triveni, Annapurna, Aathira and Samyuktha (red rice with short bold grain type), all other colored rice genotypes under study recorded slender grain type. The solid loss which affects the stability of cooked rice ranged from 0.6 (BPT 3140 ) to $2.5 \%$ (BPT 2858). Alkali spreading value (ASV) and amylose content (AC) content are the important cooking quality traits which determine the texture of cooked rice. Li et al., (2016) ${ }^{[20]}$ also stated that the amylose content of the rice variety has culinary implications because it has an influence on the organoleptic qualities of rice once cooked. Among non-pigmented rice genotypes, BPT 5204, BPT 2270, BPT 2595, BPT 2782 and BPT 2776 recorded the desirable intermediate values for both AC and ASV so that the cooked rice of these varieties will be soft and flaky. Except BPT 2858 and BPT 3111, all other red rice genotypes exhibited low values for ASV which is not desirable. Among black rice genotypes, BPT 3144, BPT 3136, BPT 3140, BPT 2848 recorded intermediate AC and ASV that gives soft and flaky texture to cooked rice. Majority of black rice varieties grown in North East Indian states are of bold \& glutinous type with low amylose content. Awadesh Kumar et al., (2018) ${ }^{[2]}$ and Sangeetha et al. (2012) ${ }^{[36]}$ also reported that the amylose content of Kalabhatt and Chakhao amubi, famous black rice varieties of North Eastern India are low $(4.27 \%$ \& $2.9 \%$ respectively). These bold and glutinous black rice varieties are not preferred in South India as the consumers prefer medium slender grain type with soft and flaky texture of cooked rice. During the last few decades, the people are more concerned about the natural health supplements from food resources. Hence, the colored genotypes possessing medium slender grain with desirable cooking quality (soft \& flaky texture of cooked rice) may be exploited commercially and can be included in daily diet for their high bioactive compounds which has potential nutraceutical benefits to health. 
Table 2: Grain yield and yield components of different colored rice (Oryza sativa L.) genotypes

\begin{tabular}{|c|c|c|c|c|c|c|c|c|c|}
\hline $\begin{array}{l}\text { S. } \\
\text { No. }\end{array}$ & Character & Days to $50 \%$ flowering & $\begin{array}{c}\text { Plant } \\
\text { height }(\mathrm{cm})\end{array}$ & \begin{tabular}{|c|} 
Ear bearing \\
tillers per plant
\end{tabular} & $\begin{array}{c}\text { Panicle } \\
\text { length }(\mathrm{cm})\end{array}$ & $\begin{array}{c}\text { Total } \\
\text { grains/panicle }\end{array}$ & $\begin{array}{c}\text { Fertility } \\
(\%)\end{array}$ & $\begin{array}{c}\text { Test weight } \\
\text { (g) }\end{array}$ & $\begin{array}{c}\text { Grain yield } \\
\text { per plant (g) }\end{array}$ \\
\hline & \multicolumn{2}{|c|}{ Brown pericarp colored genotypes } & & & & & & & \\
\hline 1 & BPT 5204 & $117 \pm 2.51$ & $88.40 \pm 1.77$ & $13.33 \pm 1.15$ & $19.89 \pm 0.64$ & $167 \pm 16.5$ & $90.5 \pm 2.03$ & $14.84 \pm 0.53$ & $25.00 \pm 3.60$ \\
\hline 2 & BPT 2270 & $127 \pm 1.52$ & $116.67 \pm 3.06$ & $15.67 \pm 2.08$ & $23.39 \pm 0.49$ & $284 \pm 14.9$ & $91.6 \pm 2.9$ & $13.81 \pm 0.46$ & $29.33 \pm 5.13$ \\
\hline 3 & BPT 2295 & $121 \pm 3.05$ & $114.73 \pm 3.16$ & $16.67 \pm 0.57$ & $27.02 \pm 1.23$ & $294 \pm 12.4$ & $89.2 \pm 1.4$ & $13.50 \pm 0.31$ & $33.33 \pm 4.16$ \\
\hline 4 & BPT 2595 & $116 \pm 1.15$ & $104.47 \pm 3.40$ & $17.00 \pm 1.0$ & $25.58 \pm 0.62$ & $276 \pm 9.0$ & $92.1 \pm 1.47$ & $14.90 \pm 0.15$ & $37.00 \pm 2.64$ \\
\hline 5 & BPT 2782 & $112 \pm 1.0$ & $94.93 \pm 2.34$ & $18.33 \pm 1.15$ & $25.39 \pm 1.12$ & $305 \pm 12.7$ & $94.0 \pm 1.21$ & $15.46 \pm 0.47$ & $47.70 \pm 1.34$ \\
\hline 6 & BPT 2660 & $21 \pm 1.0$ & $110.53 \pm 0.50$ & $16.67 \pm 0.57$ & $25.77 \pm 0.27$ & $296 \pm 18.1$ & $88.2 \pm 1.03$ & $15.31 \pm 0.54$ & $36.67 \pm 2.08$ \\
\hline \multirow[t]{2}{*}{7} & BPT 2776 & $124 \pm 3.05$ & $110.47 \pm 4.38$ & $17.67 \pm 0.57$ & $25.73 \pm 1.5$ & $297 \pm 8.1$ & $92.3 \pm 1.73$ & $14.84 \pm 0.72$ & $40.33 \pm 5.13$ \\
\hline & \multicolumn{2}{|c|}{ Red pericarp colored genotypes } & & & & & & & \\
\hline 1 & MathaTriveni & i) $\quad 103 \pm 4.35$ & $112.67 \pm 3.78$ & $14.67 \pm 2.30$ & $25.10 \pm 0.26$ & $272 \pm 13.6$ & $90.9 \pm 0.96$ & $21.09 \pm 0.77$ & $50.33 \pm 2.91$ \\
\hline 2 & Annapurna & & $110.00 \pm 2.8$ & $14.33 \pm 2.08$ & $27.44 \pm 0.94$ & $204 \pm 11.9$ & $90.5 \pm 3.28$ & $25.58 \pm 0.55$ & $45.43 \pm 4.53$ \\
\hline 3 & Aathira & & $101.93 \pm 1.81$ & $15.67 \pm 1.52$ & $25.19 \pm 2.11$ & $362 \pm 22.7$ & $86.4 \pm 2.81$ & $22.79 \pm 2.03$ & $36.13 \pm 1.58$ \\
\hline 4 & arsha & 04 & $113.13 \pm 2.80$ & $13.67 \pm 2.51$ & $27.23 \pm 2.23$ & $191 \pm 3.6$ & $92.7 \pm 0.82$ & $24.36 \pm 0.71$ & $43.53 \pm 1.68$ \\
\hline 5 & Jyothi & & $123.53 \pm 2.31$ & 15.00 & $24.47 \pm 1.35$ & $210 \pm 6.5$ & $91.4 \pm 2.54$ & $21.02 \pm 0.61$ & \pm 1.0 \\
\hline 6 & yuktha & & $123.13 \pm 1.20$ & 12.3 & $28.03 \pm$ & $209 \pm 2.1$ & $89.4 \pm 0.97$ & $90 \pm 0.80$ & 4.83 \\
\hline 7 & T 2858 & \pm 2.51 & $112.67 \pm 5.50$ & $15.00 \pm 2.0$ & $30.53 \pm 0.83$ & $391 \pm 14.6$ & $94.9 \pm 1.09$ & $13.77 \pm 0.55$ & $41.73 \pm 3.33$ \\
\hline 8 & & & $109.87 \pm 4.79$ & $13.33 \pm 1.52$ & 27.01 & $249 \pm 12.2$ & $90.4 \pm 0.30$ & $19.35 \pm 0.40$ & $42.13 \pm 0.41$ \\
\hline \multirow[t]{2}{*}{9} & BPT 3139 & $104 \pm 4.04$ & $114.67 \pm 1.92$ & $12.33 \pm 2.08$ & $28.78 \pm 2.93$ & $313 \pm 11.2$ & $91.5 \pm 01.02$ & $18.12 \pm 0.75$ & $34.90 \pm 3.2$ \\
\hline & \multicolumn{2}{|c|}{ Black pericarp colored genotypes } & & & & & & & \\
\hline 1 & & $105 \pm 4.04$ & $116.33 \pm 5.30$ & & $32.44 \pm 2.43$ & $390 \pm 9.5$ & $92.6 \pm 1.59$ & $14.30 \pm 0.37$ & $36.93 \pm 1.10$ \\
\hline 2 & BPT 3145 & \pm 3.0 & $114.27 \pm 3.88$ & $13.33 \pm 1.15$ & $30.51 \pm 0.74$ & $382 \pm 8.0$ & $94.1 \pm 1.53$ & $21.49 \pm 0.87$ & $41.77 \pm 3.45$ \\
\hline 3 & BPT 3138 & $3 \pm 3.46$ & $120.87 \pm 1.66$ & $13.00 \pm 2.0$ & $29.93 \pm 3.55$ & $393 \pm 24.0$ & $95.6 \pm 2.34$ & $19.54 \pm 0.90$ & $39.03 \pm 0.85$ \\
\hline 4 & BPT 3136 & $4 \pm 2.64$ & $105.67 \pm 4.50$ & $14.33 \pm 1.52$ & $28.64 \pm 3.11$ & $259 \pm 9.5$ & $85.9 \pm 5.05$ & $19.83 \pm 0.65$ & $48.83 \pm 4.35$ \\
\hline 5 & PT 3140 & & $116.07 \pm 4.40$ & $14.67 \pm 3.21$ & $24.92 \pm 1.13$ & $330 \pm 9.2$ & $90.9 \pm 0.58$ & $18.83 \pm 0.45$ & $39.10 \pm 1.15$ \\
\hline 6 & BPT 3141 & & $113.87 \pm 3.66$ & 13.00 & $27.21 \pm 1.67$ & $284 \pm 14.5$ & $91.5 \pm 3.12$ & $18.10 \pm 0.48$ & $37.63 \pm 3.29$ \\
\hline 7 & BPT 3142 & $3 \pm 4.50$ & $112.33 \pm 3.86$ & $14.67 \pm 1.52$ & $26.07 \pm 1.03$ & $371 \pm 21.0$ & $94.3 \pm 1.24$ & $24.31 \pm 0.49$ & $58.33 \pm 7.23$ \\
\hline 8 & BPT 3143 & $104 \pm 4.58$ & $110.80 \pm 3.70$ & $15.67 \pm 1.15$ & $28.38 \pm 2.01$ & $249 \pm 18.5$ & $93.6 \pm 1.08$ & $16.90 \pm 0.19$ & $48.03 \pm 6.47$ \\
\hline 9 & BPT 2848 & $104 \pm 3.05$ & $113.40 \pm 4.84$ & $13.67 \pm 1.52$ & $30.74 \pm 1.88$ & $417 \pm 11.9$ & $93.8 \pm 1.56$ & $14.78 \pm 0.63$ & $45.50 \pm 0.95$ \\
\hline \multirow[t]{4}{*}{10} & BPT 3144 & $107 \pm 1.52$ & $110.33 \pm 1.52$ & $15.33 \pm 1.52$ & $30.40 \pm 0.70$ & $356 \pm 7.6$ & $88.3 \pm 3.53$ & $14.60 \pm 0.20$ & $43.93 \pm 3.70$ \\
\hline & Mean & 108.52 & 111.37 & 14.68 & 27.15 & 298.78 & \begin{tabular}{|c|}
91.42 \\
\end{tabular} & 18.51 & 41.1 \\
\hline & $\mathrm{CD}(0.05)$ & 5.48 & 5.70 & 2.86 & 2.76 & 61.50 & 4.40 & 1.13 & 5.88 \\
\hline & $P<0.05$ & 0.001 & 0.001 & 0.001 & 0.001 & 0.001 & 0.001 . & 0.001 & 0.001 \\
\hline
\end{tabular}

Values in bold are minimum and maximum for each character $\mathrm{CD}=$ Critical Difference

Table 3: Physico-chemical, nutritional and functional properties of different colored rice (Oryza sativa L.) genotypes

\begin{tabular}{|c|c|c|c|c|c|c|c|c|c|c|c|c|c|}
\hline $\begin{array}{l}\text { S. } \\
\text { No. }\end{array}$ & $\begin{array}{c}\text { Designatiio } \\
\mathbf{n}\end{array}$ & $\begin{array}{c}\text { Length/ } \\
\text { Breadth } \\
\text { ratio }\end{array}$ & $\begin{array}{c}\text { Grain } \\
\text { type }\end{array}$ & Solid loss & $\begin{array}{c}\text { Volume } \\
\text { expansion } \\
\text { ratio }\end{array}$ & $\begin{array}{c}\text { Alkali } \\
\text { spreading } \\
\text { value }\end{array}$ & $\begin{array}{c}\text { Amylose } \\
\text { content } \\
(\%)\end{array}$ & $\begin{array}{l}\text { Total Phenol } \\
\text { content } \\
(\mathrm{mg} / 100 \mathrm{~g})\end{array}$ & $\begin{array}{c}\text { Total } \\
\text { Antioxidant } \\
\text { Activity (mg } \\
\text { AAE/100g) } \\
\end{array}$ & $\begin{array}{c}\text { Flavonoid } \\
\text { content } \\
(\mathrm{mg} / 100 \mathrm{~g})\end{array}$ & $\begin{array}{c}\text { Zinc } \\
\text { content } \\
(\mathbf{p p m})\end{array}$ & $\begin{array}{c}\text { Iron } \\
\text { content } \\
(\mathbf{p p m})\end{array}$ & $\begin{array}{l}\text { Protein } \\
\text { content } \\
(\%)\end{array}$ \\
\hline \multicolumn{14}{|c|}{ Brown pericarp colored genotypes } \\
\hline 1 & BPT 5204 & $2.85 \pm 0.05$ & MS & $1.1 \pm 0.08$ & $2.56 \pm 0.24$ & $5.00 \pm 0.00$ & $22.65 \pm 1.32$ & $59.40 \pm 1.68$ & $28.39 \pm 0.33$ & $144.41 \pm 11.8$ & $16.80 \pm 1.6$ & $8.70 \pm 0.6$ & $8.00 \pm 0.56$ \\
\hline 2 & BPT 2270 & $2.84 \pm 0.01$ & MS & $2.25 \pm 0.04$ & $2.25 \pm 0.02$ & $4.33 \pm 0.28$ & $20.98 \pm 0.54$ & $67.89 \pm 0.05$ & $29.94 \pm 0.30$ & $113.48 \pm 3.2$ & $15.50 \pm 1.3$ & $8.80 \pm 1.0$ & \\
\hline 3 & BPT 2295 & $2.86 \pm 0.02$ & MS & $1.35 \pm 0.02$ & $2.29 \pm 0.18$ & $3.33 \pm 0.28$ & $25.45 \pm 0.87$ & $75.59 \pm 0.23$ & $26.87 \pm 0.11$ & $128.55 \pm 18.3$ & $12.00 \pm 2.0$ & $7.90 \pm 1.8$ & $7.11 \pm 0.16$ \\
\hline 4 & BPT 2595 & $2.87 \pm 0.02$ & MS & $1.85 \pm 0.07$ & $2.00 \pm 0.14$ & $4.67 \pm 0.28$ & $23.97 \pm 0.20$ & $54.07 \pm 0.31$ & $28.31 \pm 0.46$ & $153.92 \pm 12.3$ & $15.30 \pm 1.7$ & $7.00 \pm 1.7$ & $10.21 \pm 0.2$ \\
\hline 5 & BPT 2782 & $2.82 \pm 0.03$ & MS & $1.1 \pm 0.02$ & $2.57 \pm 0.31$ & $4.33 \pm 0.28$ & $21.45 \pm 0.91$ & $69.89 \pm 0.16$ & $44.90 \pm 0.33$ & $120.68 \pm 7.9$ & $17.67 \pm 0.41$ & $10.53 \pm 1.6$ & $7.14 \pm 0.12$ \\
\hline 6 & BPT 2660 & $2.78 \pm 0.67$ & MS & $1.7 \pm 0.04$ & $2.5 \pm 0.12$ & $3.67 \pm 1.52$ & $21.11 \pm 1.28$ & & & & $18.60 \pm 3.1$ & $9.80 \pm 2.2$ & $9.83 \pm 0.18$ \\
\hline 7 & & $2.87 \pm 1.25$ & MS & $1.15 \pm 0.03$ & $2.67 \pm 0.17$ & $4.00 \pm 1.73$ & $23.55 \pm 0.74$ & $48.80 \pm 0.19$ & & & & & \\
\hline \multicolumn{14}{|c|}{ Red pericarp colored genotypes } \\
\hline 1 & $\begin{array}{c}\text { Matha } \\
\text { Triveni }\end{array}$ & $2.35 \pm 0.04$ & SB & $1.7 \pm 0.06$ & $2.25 \pm 0.19$ & $2.00 \pm 0.0$ & $27.44 \pm 0.45$ & $87.33 \pm 0.25$ & $106.44 \pm 0.43$ & $434.65 \pm 13.3$ & $25.90 \pm 1.3$ & $10.70 \pm 2.1$ & $13.54 \pm 0.12$ \\
\hline 2 & Annapurna & $2.26 \pm 0.01$ & SB & $1.7 \pm 0.03$ & $2.00 \pm 0.11$ & $3.00 \pm 0.0$ & $18.86 \pm 0.64$ & $60.25 \pm 0.13$ & $07.75 \pm 0.39$ & 296.6 & $22.00 \pm 2.0$ & $9.30 \pm 0.4$ & $9.23 \pm 0.19$ \\
\hline 3 & & $2.26 \pm 0.03$ & SB & $0.8 \pm 0.03$ & & & 24.2 & & \pm 0.34 & & & $10.70 \pm 1.8$ & \\
\hline 4 & Harsha & 2.68 & MS & $2.1 \pm$ & & & 25.3 & & & & & 12. & 13 \\
\hline 5 & Jyothi & $2.75 \pm 0.02$ & MS & $1.15 \pm 0.03$ & $2.14 \pm 0.08$ & $2.00 \pm 0.0$ & $22.0 \pm 0.13$ & 132.8 & \pm 0.28 & \pm 5.2 & 20.80 & $9.60 \pm 0.4$ & $12.48 \pm 0.12$ \\
\hline 6 & Samyuktha & $2.49 \pm 0.04$ & SB & $0.85 \pm 0.03$ & $2.00 \pm 0.18$ & $2.00 \pm 0.0$ & $26.36 \pm 0.69$ & 0.29 & $100.91 \pm 0.17$ & $290.23 \pm 5.5$ & $18.90 \pm 0.9$ & $12.50 \pm 0.9$ & $9.07 \pm 0.1$ \\
\hline 7 & BPT 2858 & $3.01 \pm 0.01$ & SS & $2.7 \pm 0.07$ & $1.86 \pm 0.15$ & $4.33 \pm 0.28$ & $21.18 \pm 0.38$ & 174.4 & $89.07 \pm 0.56$ & $548.72 \pm 7.6$ & $26.80 \pm 0.8$ & $19.40 \pm 2.2$ & $12.82 \pm 0.11$ \\
\hline 8 & BPT 3111 & $2.83 \pm 0.02$ & MS & $1.2 \pm 0.02$ & $1.75 \pm 0.12$ & $4.83 \pm 0.28$ & $23.02 \pm 0.46$ & \begin{tabular}{|l|l}
$95.50 \pm 0.17$ \\
\end{tabular} & $110.09 \pm 0.18$ & $410.61 \pm 8.9$ & $24.20 \pm 1.4$ & $16.00 \pm 1.9$ & $10.97 \pm 1.3$ \\
\hline 9 & BPT 3139 & $2.82 \pm 0.01$ & MS & $1.15 \pm 0.06$ & $2.29 \pm 0.09$ & $3.33 \pm 0.28$ & $22.4 \pm 0.28$ & $86.65 \pm 0.09$ & $105.83 \pm 0.14$ & $139.65 \pm 6.9$ & $26.20 \pm 1.7$ & $11.00 \pm 2.0$ & $7.41 \pm 0.22$ \\
\hline \multicolumn{14}{|c|}{ Black pericarp colored genotypes } \\
\hline 1 & & 2.83 & MS & .04 & & $4.00 \pm 0.0$ & & & 0.42 & & & & \\
\hline 2 & BPT 3145 & $2.85 \pm 0.03$ & MS & $2.3 \pm 0.06$ & $1.50 \pm 0.13$ & $3.67 \pm 0.28$ & $21.70 \pm 0.35$ & $125.47 \pm 0.16$ & $96.38 \pm 0.39$ & $444.36 \pm 13.1$ & $26.10 \pm 1.5$ & $10.30 \pm 0.8$ & 12.56 \\
\hline 3 & BPT 3138 & $3.08 \pm 0.07$ & LS & $1.55 \pm 0.03$ & $2.00 \pm 0.09$ & $3.00 \pm 0.50$ & $24.08 \pm 0.19$ & \begin{tabular}{|l|}
$98.48 \pm 0.27$ \\
\end{tabular} & $74.46 \pm 0.36$ & $486.98 \pm 9.7$ & $25.20 \pm 2.0$ & \begin{tabular}{|l|}
$8.40 \pm 1.2$ \\
\end{tabular} & $9.59 \pm 0.16$ \\
\hline 4 & BPT 3136 & $2.79 \pm 0.01$ & MS & $1.1 \pm 0.02$ & $2.00 \pm 0.24$ & $4.17 \pm 0.57$ & $21.12 \pm 0.74$ & $81.28 \pm 0.48$ & $73.55 \pm 0.21$ & $560.36 \pm 3.5$ & $27.00 \pm 1.1$ & $9.10 \pm 1.7$ & $13.55 \pm 0.13$ \\
\hline 5 & BPT 3140 & $3.51 \pm 0.11$ & LS & $0.6 \pm 0.06$ & $3.00 \pm 0.17$ & $4.00 \pm 0.50$ & $22.17 \pm 0.28$ & $214.34 \pm 0.25$ & $108.83 \pm 0.28$ & $590.12 \pm 7.0$ & $24.30 \pm 2.4$ & $13.40 \pm 2.3$ & $10.95 \pm 0.23$ \\
\hline 6 & BPT 3141 & $3.64 \pm 0.03$ & LS & $1.8 \pm 0.04$ & $2.25 \pm 0.07$ & $3.00 \pm 0.50$ & $20.27 \pm 0.78$ & $156.31 \pm 0.26$ & $105.57 \pm 0.26$ & $579.54 \pm 16.3$ & $27.80 \pm 0.9$ & $9.40 \pm 1.4$ & $13.39 \pm 0.08$ \\
\hline 7 & BPT 3142 & $2.70 \pm 0.06$ & MS & $1.25 \pm 0.02$ & $2.65 \pm 0.13$ & $3.00 \pm 0.0$ & $22.05 \pm 0.48$ & \begin{tabular}{|l|l}
$88.64 \pm 0.51$ \\
\end{tabular} & $77.07 \pm 0.84$ & $661.44 \pm 4.98$ & $21.80 \pm 1.1$ & $11.10 \pm 0.4$ & $13.35 \pm 0.27$ \\
\hline 8 & BPT 3143 & $2.81 \pm 0.02$ & MS & $1.9 \pm 0.06$ & $3.25 \pm 0.21$ & $3.17 \pm 0.28$ & $19.11 \pm 0.54$ & \begin{tabular}{|l|l}
$101.67 \pm 0.18$ \\
\end{tabular} & $78.36 \pm 0.18$ & $364.86 \pm 3.63$ & $27.90 \pm 0.5$ & $10.60 \pm 2.2$ & $12.45 \pm 0.06$ \\
\hline 9 & BPT 2848 & $2.82 \pm 0.02$ & MS & $2.2 \pm 0.03$ & $2.25 \pm 0.07$ & $4.17 \pm 0.57$ & $20.88 \pm 0.90$ & $123.31 \pm 0.26$ & $86.63 \pm 0.65$ & $784.54 \pm 21.6$ & 18.001 .5 & $12.30 \pm 1.4$ & $13.11 \pm 0.13$ \\
\hline 10 & BPT 3144 & $2.83 \pm 0.02$ & MS & $1.55 \pm 0.04$ & $1.50 \pm 0.12$ & $4.00 \pm 0.50$ & $22.36 \pm 0.45$ & $150.82 \pm 0.42$ & $73.01 \pm 0.61$ & $612.12 \pm 6.6$ & $19.90 \pm 2.1$ & $9.60 \pm 2.2$ & $13.52 \pm 0.17$ \\
\hline
\end{tabular}




\begin{tabular}{|c|c|c|c|c|c|c|c|c|c|c|c|c|c|}
\hline & Mean & 2.80 & - & 1.52 & 2.21 & 3.42 & 22.42 & 96.1 & 78.62 & 349.56 & 21.74 & 10.64 & 10.60 \\
\hline & CD $(0.05)$ & 0.45 & - & 0.08 & 0.26 & 0.89 & 1.11 & 0.69 & 3.58 & 18.80 & 2.60 & 2.56 & 0.31 \\
\hline & $P<0.01$ & 0.001 & - & 0.001 & 0.001 & 0.001 & 0.001 & 0.001 & 0.001 & 0.001 & 0.001 & 0.001 & 0.001 \\
\hline
\end{tabular}

LS: Long Slender; MS: Medium Slender; SB: Short Bold; SS: Short Slender Values in bold are minimum and maximum for each character

\section{Anti-oxidative and nutritional quality parameters of rice genotypes}

The results of present study revealed that black and red rice are rich in both micronutrients and protein content when compared with non-pigmented rice varieties. BPT 3141 (27.8 ppm) and BPT 3136 (27.0 ppm) recorded high $\mathrm{Zn}$ content whereas BPT 3140, BPT 2848, BPT 2858, BPT 3111, Samyuktha and Harsha are rich in Fe content. Protein is an important modulator in glucose homeostasis by increasing gluconeogenesis and preventing insulin resistance (Ke et al., 2018) ${ }^{[15]}$, hence genotypes possessing high protein content digest slowly and aids in slow release of blood glucose. In the present study, the protein content ranged from 6.23 to $13.55 \%$ and the black rice genotypes viz., BPT 3136, BPT 3140, BPT 3141, BPT 3142, BPT 3143, BPT 3144 and BPT 2848 recorded $>10 \%$ protein content. Among red rice, Matha Triveni (13.54\%) followed by Harsha (13.44\%) and BPT 2858 (12.82\%) recorded high protein content. Previously, Ahuja (2008) also reported that red and black rices are more nutritious, found to be rich in $\mathrm{Fe}, \mathrm{Zn}$, minerals and possess antioxidant properties. Chakao amudi, a famous black aromatic glutinous rice of Manipur state recorded a grain protein content of 11.07-13.20\% under different organic and inorganic sources of nutrient management (Tigangam et al., 2017) ${ }^{[45]}$.

Maximum total phenol content (TPC) was exhibited by BPT $3140(214.34 \mathrm{mg} / 100 \mathrm{~g})$ followed by BPT 2858 (174.48mg/100g), BPT 3141 (156.31 mg/100g), BPT 3144 $(150.82 \mathrm{mg} / 100 \mathrm{~g})$ and Jyothi $(132.84 \mathrm{mg} / 100 \mathrm{~g})$. Other coloured genotypes which recorded high TPC include BPT 2848 (123.31mg/100g), BPT 3145 (125.47mg/100g), When both red and black varieties were compared, the black pericarp colored rice genotypes possessed high TPC values than red rice varieties. Total antioxidant activity (AOA) and total flavonoid contents (TFC) are higher in colored rice genotypes when compared with non pigmented varieties. Yuehan et al., (2018) ${ }^{[48]}$ also reported that the free TPC of whole grain red and black rice had significantly higher values than that of white rice. The antioxidant activities of pigmented rice were compared with non pigmented rice in several studies (Nam et al. 2006; Finocchiaro et al., (2007) ${ }^{[27,5]}$ and the results demonstrated that the extracts from pigmented rice displayed higher antioxidant activity than the non pigmented rice. It is interesting to note that all red rice varieties exhibited high values for AOA than black varieties eventhough their TPC values were lower than black pericarp colored genotypes (Fig.1\&2). Finocchiaro et al., (2007) ${ }^{[5]}$ studied the radical scavenging activities of the extracts from white, black and red rice and reported that despite its less anthocyanin content, red rice contains higher antioxidant activity compared to black rice due to its proanthocyanidin content. Sangeetha et al. (2012) ${ }^{[36]}$ reported that Chakhao amubi (red rice) recorded higher antioxidant activity than Poreiton Chakhao (purple rice) inspite of high amounts of anthocyanins in purple colored Poreiton Chakhao. The reason could be the presence of oligomeric proanthocyanidin or condensed tannins in red rice (Oki et al., 2005) ${ }^{[28]}$. Among the non pigmented rice varieties, maximum flavonoid content was observed in BPT 2595 (153.92mg/100g) whereas BPT 2660 (46.67mg/100g) recorded high AOA. Black rice genotypes recorded higher values for flavonoid content than red rice genotypes. Among the black pericarp colored genotypes, BPT 2848 recorded maximum flavonoid content followed by BPT 3142 and BPT 3144. The health effects of antioxidants present in colored rice suggest potential uses of the cereal anthocyanins for positive human nutrition (Zhou, 2018) ${ }^{[50]}$ and these colored rice reduce atherosclerotic plaque by $50 \%$ more than white rice in rabbits (Ling et al., 2011) ${ }^{[22]}$.

Correlation of TPC, TFC, AOA and other nutritional traits: The correlation coefficients calculated both at phenotypic and genotypic levels between phytochemicals, antioxidant activity and nutritional parameters are given in Table 4. Total phenol content manifested significant and positive association with total antioxidant activity, flavonoid content, $\mathrm{Zn}$ and $\mathrm{Fe}$ and protein content also suggesting that the colored genotypes used in the present study are rich in micronutrients and protein content. High correlation between phenolic contents and antioxidant activity (Zhou et al., 2014; Shao et al., 2018; Yuehan et al., 2018) ${ }^{[49,48]}$, ABTS and DPPH radical scavenging activity were reported earlier in different rice samples. The study of Sytar et al. (2018) ${ }^{[43]}$ demonstrated a high correlation between antioxidant activities and content of bioactive phytochemicals (anthocyanin components cyanidin and pelargonin) in colored grain. The results of this study suggest that total phenol content, flavonoid content, total antioxidant activity are positively associated with each other and also with protein content, $\mathrm{Zn}$ and Fe content, hence simultaneous improvement of all these traits is anticipated which is useful for isolation of genotypes with beneficial health effects. The relationship of total phenol content with flavonoid content $(0.663 \& 0.664)$ and antioxidant activity $(0.445 \& 0.447)$ was positive and significant. Pathak et al. (2017) ${ }^{[29]}$ also reported similar findings. Jin et al. (2009) ${ }^{[12]}$ reported that the phenolic content was positively correlated with flavonoid content. These genotypes can be utilized in food product making for their rich phytochemicals content and high antioxidantal properties.

Table 4: Estimates of phenotypic (P) \& genotypic (G) correlation coefficients of Phenolic compounds and nutritional properties in different colored rice (Oryza sativa L.) genotypes

\begin{tabular}{|c|c|c|c|c|c|c|c|}
\hline Character & & Protein content & Total phenol content & Total antioxidant activity & Flavonoid content Zinc content & Iron content \\
\hline \multirow{2}{*}{ Protein content } & $\mathrm{P}$ & 1.000 & $0.509^{*}$ & $0.422^{*}$ & $0.777^{*}$ & $0.405^{*}$ & 0.282 \\
\cline { 2 - 8 } & $\mathrm{G}$ & 1.000 & $0.511^{*}$ & $0.425^{*}$ & $0.780^{*}$ & $0.423^{*}$ & $0.338^{*}$ \\
\hline \multirow{2}{*}{$\begin{array}{c}\text { Total phenol } \\
\text { content }\end{array}$} & $\mathrm{P}$ & & 1.000 & $0.445^{*}$ & $0.663^{*}$ & $0.424^{*}$ & $0.425^{*}$ \\
\cline { 2 - 8 } & $\mathrm{G}$ & & 1.000 & $0.447^{*}$ & $0.664^{*}$ & $0.451^{*}$ & $0.502^{*}$ \\
\hline \multirow{2}{*}{$\begin{array}{c}\text { Total antioxidant } \\
\text { activity }\end{array}$} & $\mathrm{P}$ & & & 1.000 & $0.475^{*}$ & $0.694^{*}$ & $0.456^{*}$ \\
\cline { 2 - 8 } & $\mathrm{G}$ & & & 1.000 & $0.478^{*}$ & $0.740^{*}$ & $0.532^{*}$ \\
\hline Flavonoid content & $\mathrm{P}$ & & & & .1 .000 & $0.447^{*}$ & $0.356^{*}$ \\
\hline
\end{tabular}




\begin{tabular}{|c|c|l|l|l|l|c|c|}
\hline & $\mathrm{G}$ & & & & 1.000 & $0.478^{*}$ & $0.429^{*}$ \\
\hline \multirow{2}{*}{ Zinc content } & $\mathrm{P}$ & & & & & 1.000 & $0.321^{*}$ \\
\cline { 2 - 7 } & $\mathrm{G}$ & & & & & 1.000 & $0.415^{*}$ \\
\hline \multirow{2}{*}{ Iron content } & $\mathrm{P}$ & & & & & & 1.000 \\
\cline { 2 - 8 } & $\mathrm{G}$ & & & & & & 1.000 \\
\hline
\end{tabular}

* p values significant at <0.01 P: Phenotypic correlation G: Genotypic correlation

The values in bold are highly correlated

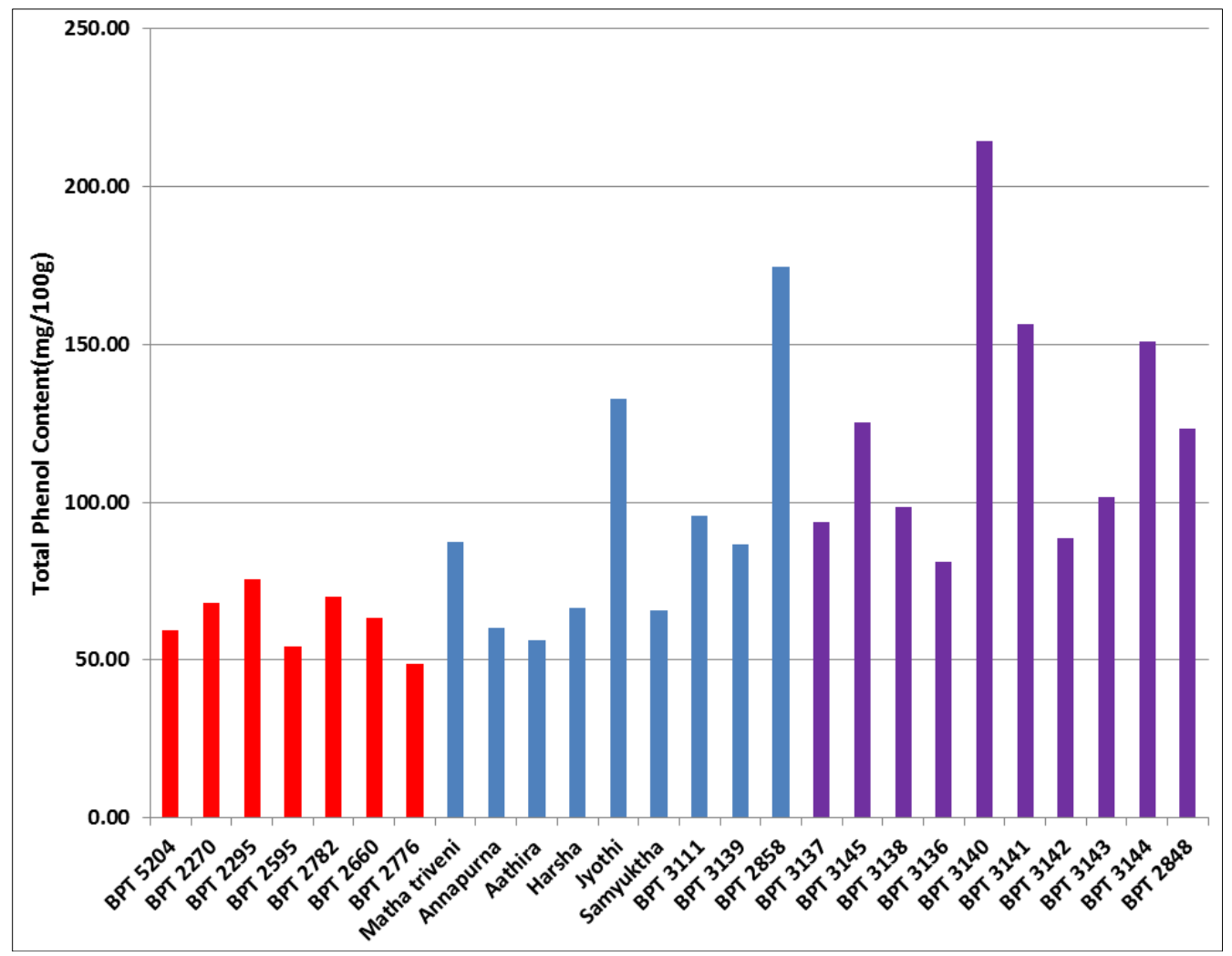

Fig 1: Total Phenol Content (mg/100g) of different colored rice genotypes

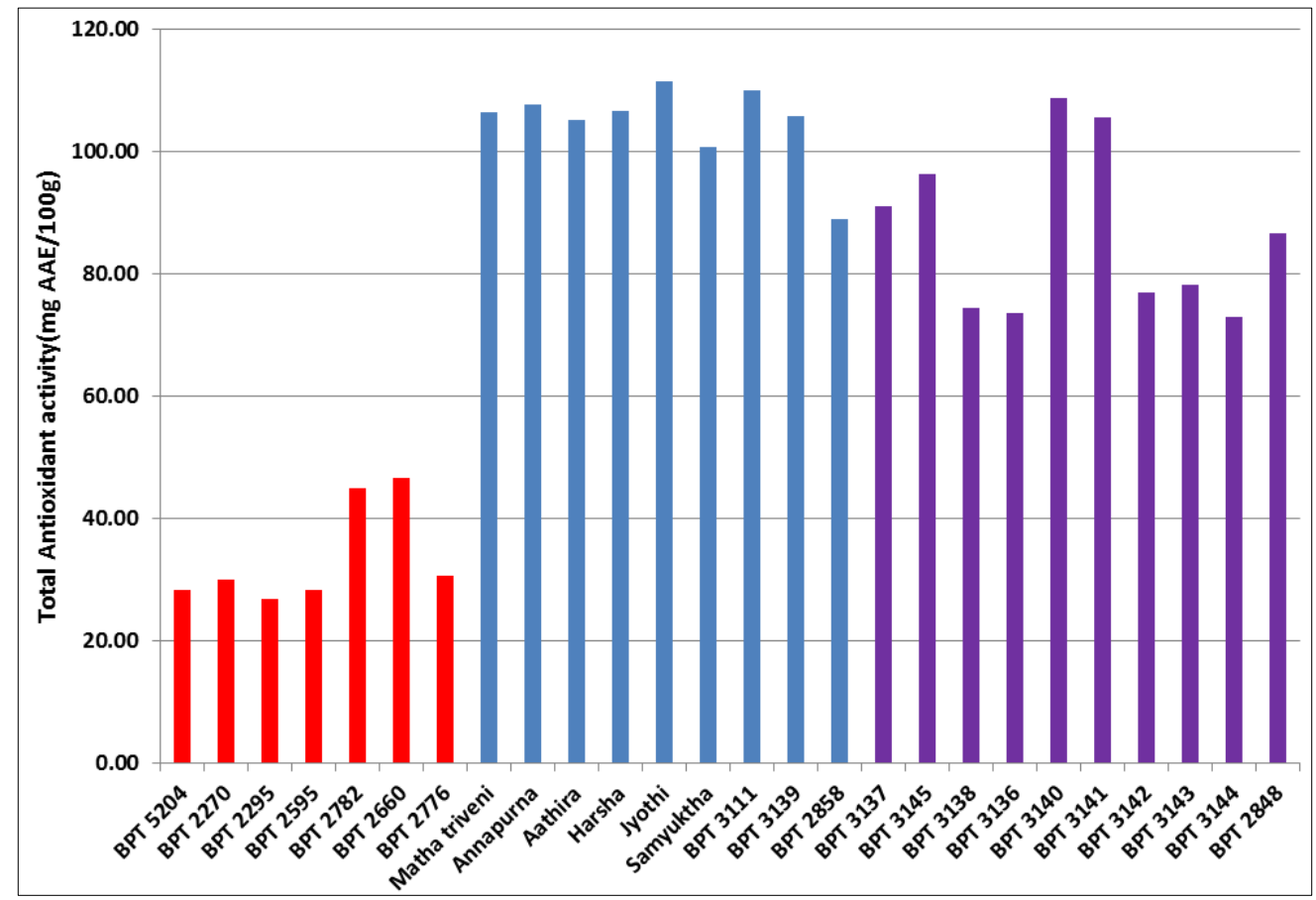

Fig 2: Total Antioxidant activity (mg AAE/100g) of different colored rice genotypes

\section{Conclusion}

The present study reveals that colored genotypes possess high TPC, total flavonoid content and AOA. Black rice genotypes viz., BPT 3140 \& BPT 2848, and BPT 2858 \& BPT 3111 among red rice recorded desirable physicochemical quality traits, high protein \& Zn content coupled with high TPC \& AOA. Hence, all these colored rice genotypes can be recommended for daily consumption in order to balance the 
free radical activity and to combat the risk of lifestyle related diseases. More publicity on the relationship between antioxidants and disease risk mechanisms would increase consumption of the anthocyanin rich colored rice.

\section{Author's contribution}

Research idea (BKV), experimental design (BKV, DPBJ), research material contributions (BKV), field and laboratory execution (DSR, PSD), data collection (PSD), analysis and interpretation of data (BKV, DSR, DPBJ), script preparation (BKV, DSR, DSD, DPBJ).

\section{Declaration}

The authors declare no conflict of interest.

\section{References}

1. Ahuja U, Ahuja SC, Rashmi Thakrar, Singh RK. Rice-A Neutraceutical. Agri-Asian History 2008;12(2):93-108.

2. Awadhesh Kumar, Upasana Sahoob, Basavdatta Baisakha, Oko Augustine Okpani, Umakanta Ngangkham, Parameswaran C et al. Resistant starch could be decisive in determining the glycemic index of rice cultivars. Journal of Cereal Science 2018;9:348-353.

3. Chowdhury BD, Anirban N, Dasgupta T. Characterization and variability analysis of rice genotypes with reference to cooking quality parameters. IOSR Journal of Agriculture and Veterinary Science 2016;9(4):8-12.

4. Devi UM, Sarawathi R, Shanthi P, Suresh R, Sanjeeva Rao D, Rajendran R. Assessment of genetic variability in traditional rice varieties based on agro- morphological traits and iron, zinc content for crop improvement. International Journal of Science and Nature 2016;7(2):244-250.

5. Finicchiaro F, Ferrari B, Glaninetti A, Dall'Asta C, Galavema G, Scazzina $\mathrm{F}$ et al. Characterization of antioxidant compounds of red and white rice and changes in total antioxidant capacity during processing. Molecular Nutrition \& Food Research 2007;51(8):1006-1019.

6. Fujiwara Y, Kono M, Ito A, Ito M. Anthocyanins in perilla plants and dried leaves. Phytochemistry 2018;147:158-166.

7. Haritha B, Vinod KK, Aarchana C, Krishnan SG, Bhowmick PK, Nagarajan M et al. Characterising the diversity of grain nutritional and physico-chemical quality in Indian rice landraces by multivariate genetic analyse. Indian Journal of Genetics and Plant Breeding 2020;80(1):26-38.

8. Hodge JE, Hofrieter BT. In: Methods in carbohydrate chemistry. Academic press. Newyork 1962.

9. Hossain PMD, Kawar BMD, Nahas ME. Obesity and diabetes in the developing world- a growing challenge. The New England Journal of Medicine 2007;356:213215.

10. Indian Institute of Rice Research (IIRR, Hyderabad). Laboratory Manual on Rice Grain Quality Procedures 2006.

11. Isnaini $\mathrm{C}$, Pattavara Pathomrungsiyounggui, Nattaya Konsue. Effect of preparation method on chemical property of different Thai rice variety. Journal of Food and Nutrition Research 2019;7(3):231-236.

12. Jin L, Shen Y, Xiao P, Lu Y, Ba J. Total phenolics flavonoids antioxidant capacity in rice grain and their relations to grain color size and weight. Journal of Cereal Science 2009;49:106-111.
13. Johnson HW, Robinson HF, Comstock RE. Estimation of genetic and environmental variability in soybean. Agronomy Journal 1955;47:314-318.

14. Juliano BO. A simplified assay for milled rice amylose. Cereal Science Today 1971;16:334-338.

15. Ke Q, Chen C, He F, Ye Y, Bai X, Cai L. Association between dietary protein intake and type 2 diabetes varies by dietary pattern. Diabetol Metab Syndr 2018, 10.

16. Kennedy G, Burlingame B, Nguyen VN. Nutritional contribution of rice and impact of biotechnology and biodiversity in rice-consuming countries. Food and Agriculture Organization of the United Nations 2019.

17. Khoo HE, Azrina Azlan, Sou Teng Tang, See Meng Lim. Anthocyanidins and anthocyanins: colored pigments as food, pharmaceutical ingredients and the potential health benefits. Food \& Nutrition Research 2017;61:1.

18. Kong S, Kim DJ, Oh SK, Choi JS, Jeong HS, Lee J. Black rice bran as ingradient in noodles, chemical and functional evaluation. Journal of Food Science 2012;77(3):303-307.

19. Lakshmi L, Rao MVB, Raju Ch.S, Reddy SN. Variability, correlation and path analysis in advanced generation of aromatic rice. International Journal of Current Microbiology and Applied Sciences 2017;6(7):1798-1806.

20. Li H, Prakash S, Nicholson TM, Fitzgerald MA, Gilbert RG. The importance of amylose and amylopectin fine structure for textural properties of cooked rice grains. Food Chemistry 2016;196:702-711.

21. Li Y, Dongyun Ma, Dexiang Sun, Chenyang Wang, Jian Zhang, Yingxin Xie, Tiancai G. Total phenolic, flavonoid content and antioxidant activity of flour, noodles, and steamed bread made from different colored wheat grains by three milling methods. The Crop Journal 2015;3(4):328-334.

22. Ling WH, Cheng QX, Ma J, Wang T. Red and black rice decrease atherosclerotic plaque formation and increase antioxidant status in rabbits. Journal of Nutrition 2011;131:1421-1426.

23. Little RR, Hilder GB, Dawson EH, Elsie H. Differential effect of dilute alkali on 25 varieties of milled white rice. Cereal Chemistry 1958;35:111-126.

24. Lowry OH, Rosebrough NG, Far AL, Randall RJ. Protein measurement with the folin phenol reagent. Journal of Biological Chemistry 1951;193:265-275.

25. Malik CP, Singh MB. In: Plant Enzymology and Histo Enzymology, Kalyani publishers, New Delhi 1980.

26. Mamata K, Rajanna MP, Savita SK. Assessment of genetic parameters for yield and its related traits in $F_{2}$ populations involving traditional varieties of rice (Oryza sativa L.). International Journal of Current Microbiology and Applied Sciences 2018;7(1):2210-2217.

27. Nam SH, Choi SP, Kang MY, Koh HJ, Kozukue N, Friedman M. Antioxidative activities of bran extracts from twenty one pigmented rice cultivars. Food Chem 2006;4:613-620.

28. Oki T, Masuda M, Nagai S, Takeichi M, Kobyashi M, Nishiba Y. Radical scavenging activity of red and black rice. Proceedings of the World Rice Research Conference 2005, 256-259.

29. Pathak K, Rahman SW, Bhagawati S, Bhabesh G. Assessment of nutritive and antioxidant properties of some indigenous pigmented hill rice (Oryza sativa L.) cultivars of Assam. Indian Journal of Agricultural Research 2017;51(3):214-220. 
30. Pathirana CML, Shahidi F. Antioxidant properties of commercial soft and hard winter wheats (Triticum aestivum L.) and their milling fractions. Journal of the Science of Food and Agriculture 2005;86:477-485.

31. Patil CA, Kumar SN, Rajanna MP, Swamy HVVK, Ashok TH, Shashidhar HE. Micronutrient (Zinc and Iron) productivity in rice (Oryza sativa L.). Ecology Environment and Conservation Journal 2015;21:337-346.

32. Patindol JA, Guraya HS, Champagne ET, Anna MM. Nutritionally important starch fractions of rice cultivars grown in southern united states. Journal of Food Science 2010;75(5):137-144.

33. Purwanto EW, Hidayati, Nandariyah. The yield and quality of black rice varieties in different altitude. IOP Conf. Series: Earth and Environmental Science 2018, 142.

34. Raja TB, Asif M, Mallikarjun SK, Prasad SG. Variability, heritability and genetic advance for yield and yield attributing characters in different local rice (Oryza sativa L.) cultivars. Asian Journal of Bio Science 2013;8(1):60-62.

35. Sameera SK, Srinivas T, Rajesh AP, Lakshmi VJ, Nirmala PJ. Variability and path co-efficient for yield and yield components in rice. Bangladesh Journal of Agricultural Research 2016;41(2):259-271.

36. Sangeeta Sai KIA, Himjyothi Dutta, Daizi Saikia, Charu Lata Mahanta. Quality characterization and estimation of phytochemicals content and antioxidant capacity of aromatic pigmented and non-pigmented rice varieties. Food Research International 2012;46:334-340.

37. Shao YF, Bao JS. Polyphenols in whole rice grain: genetic diversity and health benefits. Food Chemistry 2015;180:86-97.

38. Shao YZ, Hu Y, Yu R, Mou Z, Zhu, Beta T. Phenolic acids, anthocyanins, proanthocyanidins, antioxidant activity, minerals and their correlations in nonpigmented, red, and black rice. Food Chem 2018;239:733-741.

39. Sidhu JS, Gill MS, Bains GS. Milling of paddy in relation to yield and Quality of Agricultural and Food chemistry 1975;23(6):1183-1185.

40. Somsana P, Wattana P, Suriharn B, Sanitchon J. Stability and genotype by environment interactions for grain anthocyanin content of Thai black glutinous upland rice (Oryza sativa. L). SABRAO journal of Breeding and Genetics 2013;45(3):523-532.

41. Subramaniam S, Menon M. Inheritance of short stature in rice. Madras Agriculture Journal 1973;60:1129-1133.

42. Sunayana R, Yadav RNS, Sarma RN. Variability in grain quality characters of upland rice of Assam, India. Rice Science 2010;17(4):330-333.

43. Sytar O, Bosko P, Zivcak M, Brestic M, Smetanska I. Bioactive phytochemicals and antioxidant properties of the grain and sprouts of colored wheat genotypes. Molecules 2018;23:2282.

44. Tian S, Nakanura K, Kayahara H. Analysis of phenolic compounds in white rice brown rice and germinated brown rice. Journal of Agricultural and Food Chemistry 2004;52:4808-4813.

45. Tigangam P, Gangmei, George PJ. Black rice CV. 'Chakhao Amubi' (Oryza sativa L.) Response to organic and inorganic sources of nutrients on growth, yield and grain protein content. Journal of Pharmacognosy and Phytochemistry 2017;6(4):550-555.
46. Uphoff M. Rice as a source of nutrition and health. International Rice Meeting 2008.

47. Yawadio R, Tanimori S, Morita N. Identification of phenolic compounds isolated from pigmented rice and their aldose reductase inhibitory activities. Food Chemistry 2007;101:1616-1625.

48. Yuehan Pang, Sulaiman Ahmed, Yanjie Xu, Trust Beta, Zhiwei Zhu, Yafang Shao et al. Bound phenolic compounds and antioxidant properties of whole grain and bran of white, red and black rice. Food Chemistry 2018;240:212-221.

49. Zhou Z, Chen X, Zhang M, Blanchard C. Phenolics, flavonoids, proanthocyanidin and antioxidant activity of brown rice with different pericarp colors following storage. Journal of Stored Products Research 2014;59:120-125.

50. Zhou F. Anthocyanins in cereals: Composition and health effects. Food Research International 2018;109:232-249. 